\title{
Proyecto "La Casa de Tod@s" utilización del medio audiovisual para exponer problemáticas de género en comunidades minoritarias en las zonas con necesidad de transformación social.
}

\author{
Project "The House of All" use of the audivisual environment to expose gender problems in \\ minority communities in the areas with the need of social transformation.
}

\begin{abstract}
Projete "A Casa de Todos" o uso do ambiente audisivual para experimentar problemas de gênero em comunidades minoríveis nas áreas com a necessidade de transformação social.
\end{abstract}

Dra. Gloria Santiago Méndez: gloria.santiago@uma.es.Universidad de Málaga

D. Antonio Villanueva Martín: antoniovillanuevamartin@gmail.com. Universidad de Málaga. Coordinador del proyecto La Casa de Tod@s y realizador audiovisual de la Trilogía de La Palmilla

Resumen: Proyecto de integración de la población inmigrante en el distrito de PalmaPalmilla (Málaga) en el entorno de una asociación gitana durante los años posteriores a la crisis del 2007/2008 fomentando la participación activa y la difusión de la figura del voluntariado con tres ejes fundamentales: la educación, la visibilidad y la igualdad.

Metodología: Tomamos dos modelos teóricos de trabajo:

La mediación entre iguales. La educación a través de Mediadores Sociales (educación inter pares) como estrategia para el cambio del comportamiento se basa, entre otras, en la Teoría del aprendizaje social (Bandura, 1986), o en la Teoría de la acción razonada (Fishbein \& Ajzen, 1975), y que se importa a través del conocimiento que se tiene del "Proyecto Bola de Nieve. Guía para la formación de usuarios de la droga como agentes de salud" (García, Gutiérrez y Morante, 2004) que lo sintetiza previamente.

Iniciación de un proceso de empoderamiento de mujeres gitanas, inmigrantes y ex presidiarias. Este proyecto tiene algún remoto precedente teórico en el movimiento de mujeres musulmanas francesas "Ni putas ni sumisas". Se actúa desde la matriculación de niñas musulmanas en institutos, la intervención en foros de participación del barrio posicionándose en temas de maltrato, machismo, racismo o derecho al trabajo, y atribuyendo sonoridad a las nuevas lideresas Ani Cortés (gitana), Yuli Fajardo (ex presidiaria), Cristiana (subsahariana), Fati ElHachimi(marroquí) y otras surgidas en hábitats patriarcales.

Conclusiones: se inicia un proyecto audiovisual para intentar plasmar lo que allí está ocurriendo difundiéndolo a través de las redes sociales y de una trilogía de cortometrajes (2008-2015) www.la trilogiadelapalmilla.blogspot.com:

* Ruta del cole 2009 (tema la educación)

* El edificio fantasma 2010 (la visibilidad)

* Vida desatenta 2015 (la superación)

Palabras Claves: Interculturalidad; lideresas; inmigrante; comunidad gitana; barrios olvidados; mediación; audiovisual

Abstract: Project to integrate the immigrants in the district of Palma Palmilla (Málaga) in the context of a gipsy association during the years following the 2007/2008 crisis, to active participation and dissemination of the figure of volunteering with three keys fundamentals: education, visibility and equality.

Methodology: We take two theoretical models of work: 
* Mediation between equals. Education through Social Mediators as a strategy for behavior change is based, among others, on the Theory of social learning (Bandura, 1986), or on the Theory of reasoned action (Fishbein \& Ajzen, 1975), and that is imported through the knowledge of the "Snowball Project. Guide for the training of users of the drug as health agents "(García, Gutiérrez and Morante, 2004) that synthesizes previously.

* Initiation of a process of empowerment of gypsy women, immigrants and former inmates. This project has some remote theoretical precedent in the movement of French Muslim women "No whores or submissive." Acting from the enrollment of Muslim girls in high schools, the intervention in neighborhood participation forums positioned on issues of abuse, machismo, racism or right to work, and attributing sound to the new women leaders Ani Cortés (gypsy), Yuli Fajardo (former inmate), Christian (sub-Sahara), Fati ElHachimi (Moroccan) and others emerged in patriarchal habitats.

Conclusions an audiovisual project is started to try to capture what is happening there by spreading it through social networks and a trilogy of short films (2008-2015) www.la trilogiadelapalmilla.blogspot.com:

* Ruta del cole 2009 (education theme).

* Edificio Fantasma 2010 (visibility)

* Vida desatenta 2015 (overcoming)

Keywords: Interculturality; women leaders; immigrant; gypsy community; neighborhoods forgotten; mediation; audiovisual

Resumo: Projeto para integrar a população imigrante no distrito de PalmaPalmilla (Málaga) no cenário de uma associação Roma durante os anos que se seguiram à crise de 2007/2008, incentivando a participação ativa e a divulgação da figura de voluntariado com três eixos Fundamentos: educação, visibilidade e igualdade.

Metodologia: Tomamos dois modelos teóricos de trabalho:

Mediação entre iguais. A educação através dos mediadores sociais (educação paritária) como estratégia de mudança comportamental baseia-se, entre outros, na Teoria da aprendizagem social (Bandura, 1986) ou na Teoria da ação racional (Fishbein \& Ajzen, 1975), e isso é importado através do conhecimento do "Projeto Snowball. Guia para o treinamento de usuários da droga como agentes de saúde "(García, Gutiérrez e Morante, 2004) que sintetiza anteriormente.

Iniciação de um processo de empoderamento de mulheres romanichéis, imigrantes e antigos detentos. Este projeto tem algum precedente teórico remoto no movimento de mulheres muçulmanas francesas "Sem prostitutas ou submissas". Atuando a partir da matrícula de meninas muçulmanas nas escolas secundárias, a intervenção em fóruns de participação de vizinhança posicionados em questões de abuso, machismo, racismo ou Direito ao trabalho e atribuição de som aos novos líderes Ani Cortés (cigano), Yuli Fajardo (antigo preso), cristão (sub-sahariano), Fati ElHachimi (marroquino) e outros surgiram em habitats patriarcais.

Conclusões: um projeto audiovisual começa a tentar capturar o que está acontecendo lá espalhando-o através de redes sociais e uma trilogia de curtas-metragens (2008-2015) www.la trilogiadelapalmilla.blogspot.com:

* Ruta del cole 2009 (tema educacional)

* O edifício fantasma 2010 (visibilidade)

* Vida desatenta 2015 (a superação)

Palavras-chave: interculturalidade; líderes; imigrante comunidade cigana; bairros esquecidos; mediação audiovisual 
1. Introducción: Proyecto de integración de la población inmigrante en el distrito de PalmaPalmilla (Málaga) en el entorno de una asociación gitana durante los años posteriores a la crisis del 2007/2008 fomentando la participación activa y la difusión de la figura del voluntariado con tres ejes fundamentales: la educación, la visibilidad y la igualdad.

En un entorno condicionado por la incidencia de las drogas en las décadas anteriores (que han generado prisión, enfermedad y muerte) Ana Cortés y Jesús Rodríguez fundan, concienciado por tanta perdida, en 2007 una asociación en su barrio de Palma Palmilla: la Asociación de Integración de la Comunidad Gitana de la Palma Palmilla. En ese territorio se acaban asentando numerosa población inmigrante (predominantemente marroquí, rumana y nigeriana) ante la imposibilidad de poder alquilar en otros lugares dónde piden garantías de las que ellos carecen. Acaban aunando esfuerzos (en un entorno donde es frecuente el conflicto intercultural) en proyectos encaminados ayudar a los sectores en mayor riesgo de exclusión: indigentes, mujeres y niños

\section{Metodología: Tomamos dos modelos teóricos de trabajo:}

2.1 La mediación entre iguales. La educación a través de Mediadores Sociales (educación inter pares) como estrategia para el cambio del comportamiento se basa, entre otras, en la Teoría del aprendizaje social (Bandura, 1986), o en la Teoría de la acción razonada (Fishbein \& Ajzen, 1975), y que se importa a través del conocimiento que se tiene del "Proyecto Bola de Nieve. Guía para la formación de usuarios de la droga como agentes de salud" (García, Gutiérrez y Morante, 2004) que lo sintetiza previamente. El proyecto Bola de Nieve fue conocido a través del Trabajador Social del Ayuntamiento de Málaga Fernando Fernández de Castro "Nando" y de Juan Manuel García Piñero director en aquel momento de los servicios sociales del distrito Palma Palmilla y fue implantado originariamente en la Casa de la Buena Vida, centro social que trabajaba la exclusión social y la indigencia y la prevención de la misma en el distrito Palma Palmilla fundado en septiembre del 2008. "La operación Bola de Nieve" del Doctor Jean-Pierre Jacque, consiste en reclutar a (ex)-UD para llevar a cabo una labor de prevención de proximidad, con el fin de que hablen con otros usuarios, sobre el Sida, las hepatitis y de los distintos modos de protegerse".

La Casa de la Buena Vida era un proyecto más plural en el que confluyeron desde ex toxicómanos y ex presidiarios, a inmigrantes, enfermos venidos de hospitales e incluso mujeres maltratadas. Por tanto la adaptación práctica del proyecto originario sufrió numerosos 
cambios hasta mutar en uno original aunque siempre estructurado sobre la base de la mediación entre iguales.

\subsection{Iniciación de un proceso de empoderamiento de mujeres gitanas, inmigrantes y ex} presidiarias. Este proyecto tiene algún remoto precedente teórico en el movimiento de mujeres musulmanas francesas "Ni putas ni sumisas" promovido por la activista Fadéla Amara que se centra en la lucha de las mujeres musulmanes de las barriadas obreras. Se actúa desde la matriculación de niñas musulmanas en institutos, la intervención en foros de participación del barrio posicionándose en temas de maltrato, machismo, racismo o derecho al trabajo, y atribuyendo sonoridad a las nuevas lideresas Ani Cortés (gitana), Yuli Fajardo (ex presidiaria), Cristiana (subsahariana), Fati ElHachimi (marroquí) y otras surgidas en hábitats patriarcales. Dicho proyecto estuvo a punto de plasmarse en una asociación de mujeres de distintas culturas junto con la activista pro derechos de la diversidad funcional Trini Recio, que en aquel momento trabajaba en el Área de Política Social del sindicato Comisiones Obreras de Málaga. Finalmente no ocurrió la creación de dicha asociación, sin embargo, cada una de ellas tuvo una trayectoria a nivel individual .Ana Cortés, tras abandonar la Asociación de Integración de la Comunidad Gitana de la Palma Palmilla que había co-fundado, llegó a ir a las listas electorales de Unidad Popular por la circunscripción de Málaga y fue representante en el distrito Centro del grupo municipal Málaga para la Gente. Cristiana, involucrada de forma tangencial en el proyecto ,acabó fundando una asociación de mujeres nigerianas. Yuli Fajardo terminó integrándose en el movimiento okupa y fue presidente durante unos meses de la Asociación Okupa tus derechos. Fátima el Hachimi optó por hacerse voluntaria en Cruz Roja de Málaga, teniendo un papel muy significativo en la lucha por los derechos de los refugiados debido a su conocimiento nativo de la lengua árabe y francesa. Siendo nombradas en el II Congreso Estatal de Voluntariado de Cruz Roja celebrado en Valencia en febrero del 2016 como una de las 3 mejores voluntarias de Cruz Roja Española en el año anterior por su labor sobre todo con los refugiados sirios llegados a Málaga. 


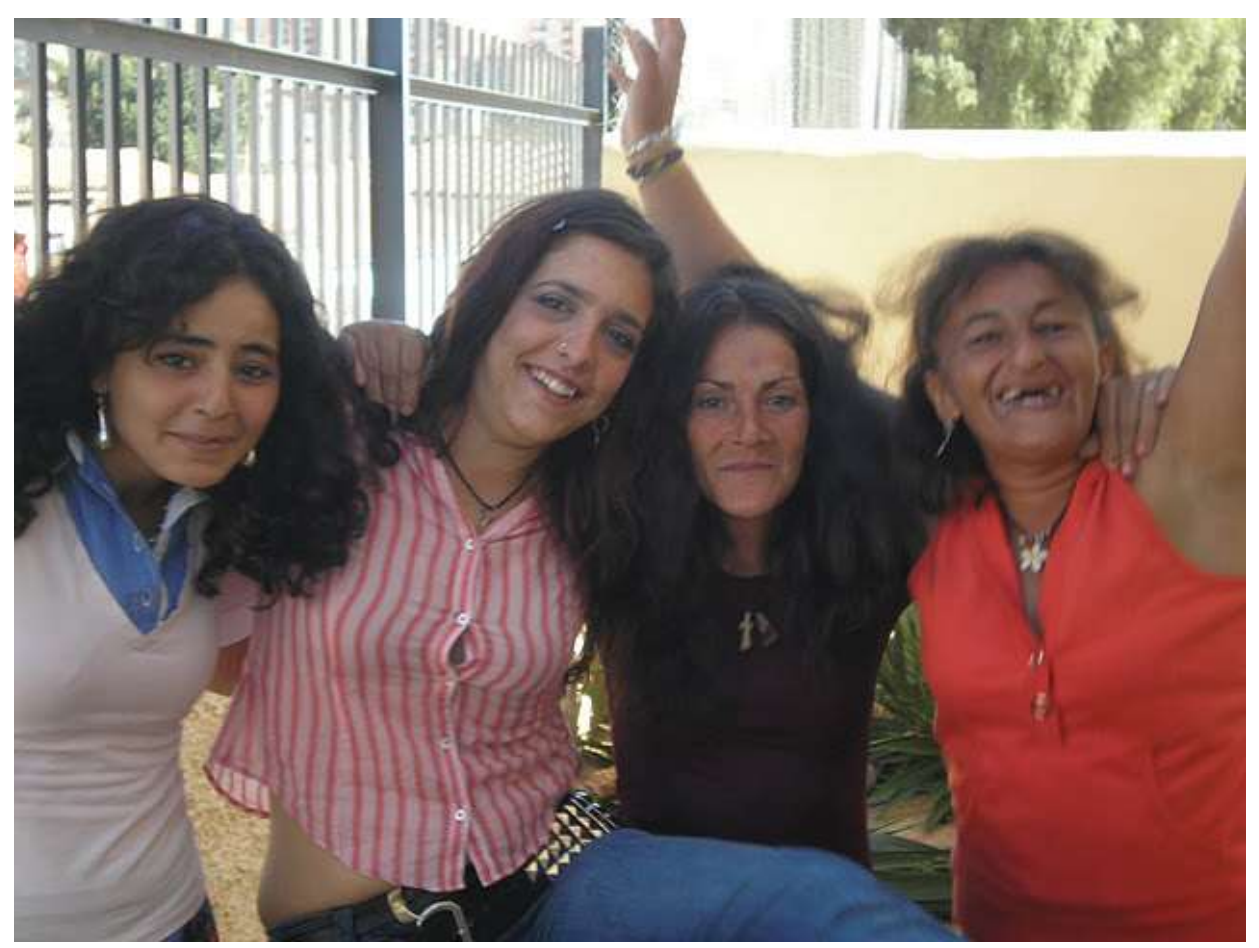

Fuente: Elaboración propia (2009)

3. Visibilización: se inicia un proyecto audiovisual para intentar plasmar lo que allí está ocurriendo difundiendo a través de las redes sociales y de una trilogía de cortometrajes (20082015).

3.1 La Ruta del Cole. Parte Primera de la Trilogía (Premio RTVA Festival Tikinó Abril 2009; Finalista Mejor Corto Documental

3.2 El Edificio Fantasma Parte Segunda de la Trilogía Finalista Mejor Corto Documental Andaluz-Diputación de Málaga, Junio 2010.

3.3 Vida Desatenta Parte Tercera de la Trilogía Accésit Tikinó noviembre 2015 www.la trilogiadelapalmilla.blogspot.com:

\section{Resumen La Trilogía de la Palmilla}

\begin{tabular}{|c|c|c|c|c|}
\hline \multicolumn{5}{|c|}{ La Trilogía de la Palmilla } \\
\hline Parte & Nombre & Año Rodaje & Año Producción & Minutos \\
\hline $1^{\mathbf{o}}$ & La Ruta del Cole & 2008 & 2009 & 11 \\
\hline $2^{\mathbf{o}}$ & El Edificio Fantasma & 2009 & 2010 & 7 \\
\hline $3^{\mathbf{0}}$ & Vida Desatenta & $2011,2012,2014$ & 2015 & 7 \\
\hline
\end{tabular}

Fuente: Elaboración propia (2018) 
3.1.1: Ruta del cole 2009 (tema la educación). El matrimonio formado por Ana y Jesús fundan la Asociación de Integración para la Comunidad Gitana de la Palma-Palmilla con el propósito, entre otros, de intentar acabar con el absentismo escolar en el barrio malagueño de Palma-Palmilla, conocido por su mala fama y su degradación social.

'La Ruta del Cole' es un documental hiperrealista que persigue a los mediadores de la Asociación de Integración que a modo de los conductores de autobuses escolares hacen La Ruta del Cole.

\section{Guión y Dirección Antonio Villanueva}

Intervienen: Jesús Rodríguez, Ana Cortés, José Arenzana, Jesulillo Rodríguez Cortés

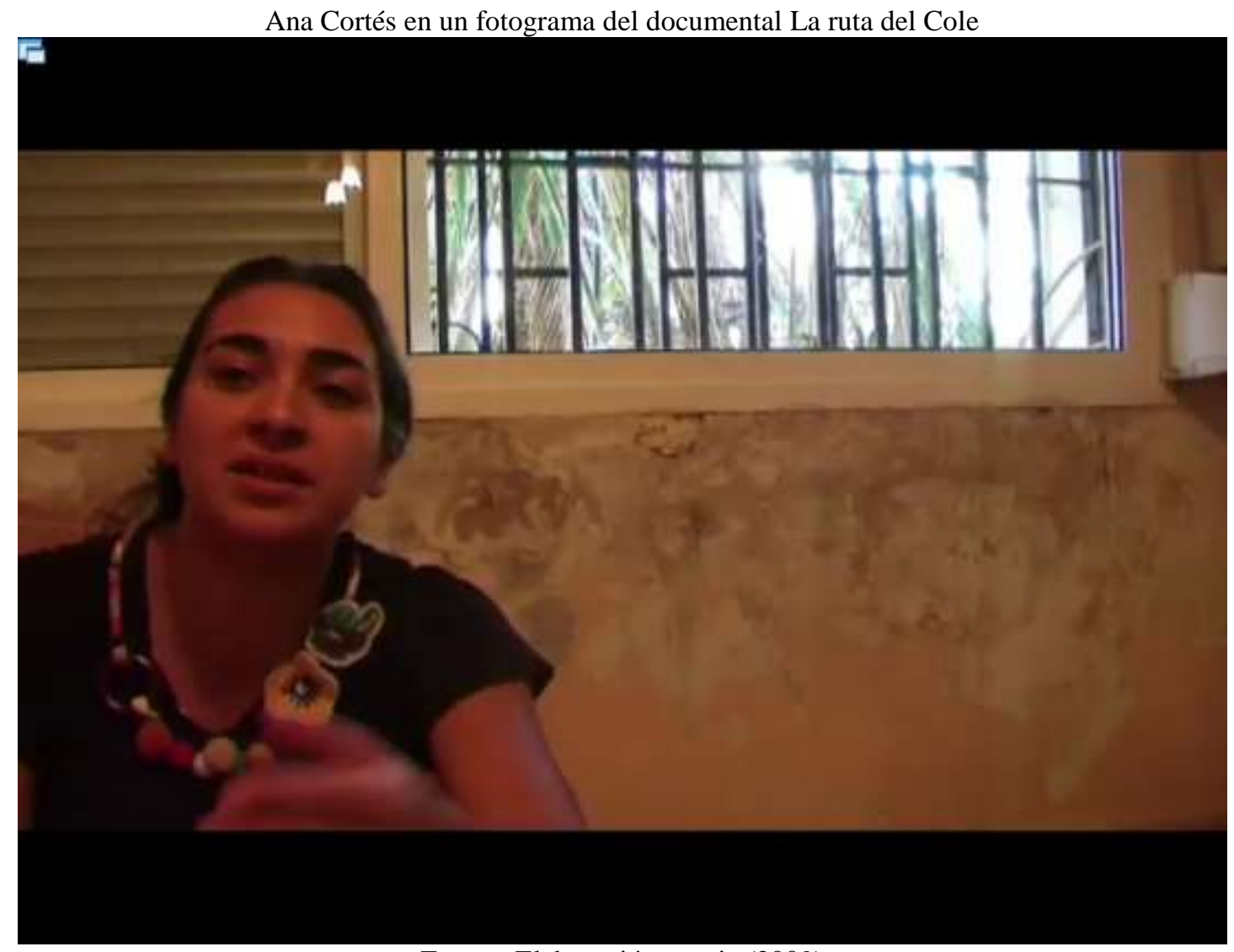

Fuente: Elaboración propia (2009)

3.2.1 : El edificio fantasma 2010 (la visibilidad) Pablo, voluntario de una asociación de la Palmilla, evoca a dos niños gemelos rumanos gitanos de 10 años(Daniel y Lorenzo) que vivieron en el edificio de Calle Cabriel 27 de trece plantas, reconocido en los medios de comunicación por su deterioro. Para los niños el bloque en el que residían en la quinta planta sin ascensor y sin luz es 'El Edificio Fantasma'

\section{Guión y Dirección Antonio Villanueva}

Intervienen: Daniel Caldaras, Lorenzo Caldaras, Pablo Cortés, Jesús Rodríguez 
3.3.1 Vida desatenta 2015 (la superación) Fati es una joven marroquí de 26 años enferma de Crohn residente en el barrio malagueño de Palma-Palmilla. A pesar de colaborar como voluntaria en numerosas asociaciones, no consigue regularizar su situación en España con un trabajo y teme, por ello, perder las prestaciones sanitarias que tiene. Su historia es la de una incansable luchadora que intenta superar su enfermedad e integrarse en la sociedad que la ha acogido.

\section{Guión y Dirección Antonio Villanueva}

Intervienen: Fatima El Hachimi, Leon Pecasse, Antonio Ruíz, Yuli Fajardo, Gloria Santiago

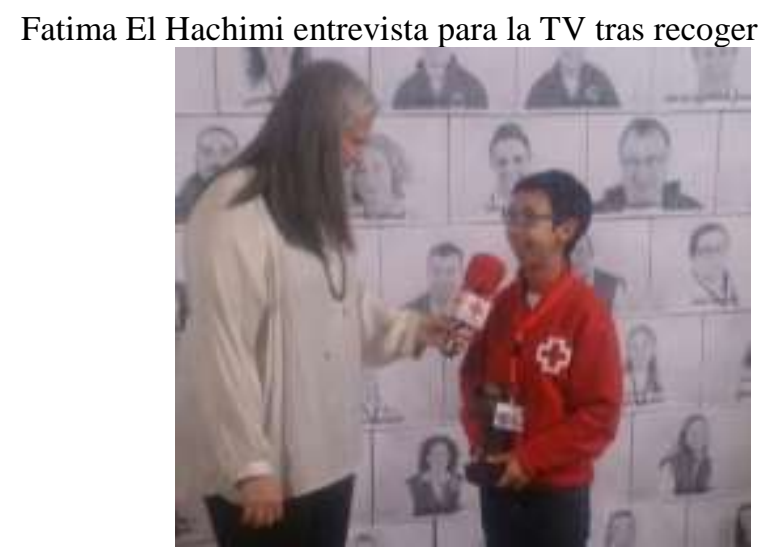

Fuente: Elaboración propia (2016)

Dentro del proyecto audiovisual también se enmarca el largometraje documental codirigido por Antonio Villanueva y Carlos Rodríguez titulado Al peor de todos los hombres ,sobre la experiencia de vida de alguno de los componentes de La casa de la buena vida de Málaga estrenado en el Festival de Cine y Derechos Humanos de Barcelona/NY/ París en diciembre del 2016 .Sinopsis: En la década de los ochentas, recién llegada la democracia a España, unos adolescentes, apenas niños, empiezan en el consumo temprano de todo tipo de drogas. Los barrios periféricos de las grandes ciudades se convierten en su feudo, en el cual ni la policía se atreve a entrar. Treinta años después ,un grupo de supervivientes crea La Casa de la Buena Vida en Málaga

Asimismo se opta por dar difusión a través de las redes sociales. Fundamentalmente se actúa a través de un canal de Facebook, un blog de Blogger y YouTube bajo la denominación gitanos Palma Palmilla. El blog se crea en febrero 2008 y a continuación los otros canales que 
estuvieron activos hasta los primeros meses del 2013. Por razones que se desconocen tanto el blog como el canal de YouTube fueron eliminados.

En 2008/2009 se colaboró de manera espasmódica con la radio comunitaria Onda Color con el programa Esperando a que empiece mi vida, reivindicando los derechos de la mujer en comunidades minoritarias (107.3 FM).

Aprovechando la actividad profesional de Antonio Villanueva cómo filmmaker, se fue creando un archivo documental audiovisual de las distintas actividades de la asociación: conciertos benéficos, semana del Gitano andaluz, día de la inmigración, día contra el racismo recitales de poesía multilingüe Carrera Popular San Silvestre Palma Palmilla y un largo etcétera. El Día contra el Racismo se coorganizó junto al sindicato Comisiones Obreras con el que se firmó un convenio en noviembre del 2010 en el que ya se explícita la necesidad de utilizar las artes audiovisuales y las redes sociales para difundir los proyectos que se estaban poniendo en marcha con especial mención a potenciar políticas que disminuyan la desigualdad:

"Cláusula VII. Difundir utilizando las artes audiovisuales y las nuevas tecnologías para promocionar las actuaciones realizadas haciendo una labor de comunicación para vencer barreras culturales basadas en el desconocimiento y en el recelo del ajeno Cláusula VIII. Animar a promover campañas en favor de la maternidad/paternidad responsable, el respeto por las minorías, la desaprobación de escenarios de injusticia hacia la mujer".

\section{Conclusiones}

El proyecto estuvo activo durante los años $(2008$ - 2011). A partir de esa fecha los distintos componentes que habían participado optaron por desarrollar otros proyectos individuales o adhiriéndose a otras organizaciones, aunque siguieron teniendo contactos durante los años cómo se explícita a lo largo de la comunicación.

Como proyecto fututo, planteamos extender las experiencias vividas durante estos años y que puedan ayudar a otras comunidades en la misma situación.

\section{Bibliografía}

Ajzen, L \& Fishbein, M. (1980) Undestandding attitudes and predicting social behavior. Englewood Cliffs, NJ: Prentice - Hall

Amara Fadela. (2004) Ni putas ni Sumisas, ES. Catedra 
Bandura, Albert. (1987) Teoría del aprendizaje social. Madrid: Espasa Libros.

Chomsky, Noam Y Dieterich, Heinz (2000): La aldea global. Navarra Tafalia: Txalaparta.

Gálvez Muñoz, Lina y Torres „Juan (2010) Desiguales: Mujeres y hombres en la crisis financiera. Barcelona: Icaria

García Galinde, Gloria, Gutierrez Hernanz, Eva y Morante Saboya, Luis. (2004) Bola de Nieve: Guía para la formación de usuarios/as de drogas como agente de salud. Madrid. Grupo GID

Navarro, Vicent (2007) La situación social en España volumen 2. Barcelona. Programa en Políticas Públicas y Sociales-Universidad Pompeu Fabra, Fundación Francisco Largo Caballero y Biblioteca Nueva

Rogers, E. M. (1983). Diffusion of innovations (3rd ed.). New York: Free Press.

Romero, José María. (2009) Maneras de hacer: propuestas para Palma Palmilla. Universidad de Granada

Rubio Diaz, Alfredo. (1979) Algunos aspectos del núcleo urbano Palma- Palmilla, de Málaga, 1959- 1975. Facultad de Filosofía y Letras. Universidad de Málaga

Villanueva Martín, Antonio (2011) Historias de La Palmilla. Editorial Airon

\section{Agradecimientos}

Don Juan Manuel García Piñero (Ayuntamiento de Málaga), Don Fernando Fernández de Castro (Ayuntamiento de Málaga), Doña Trinidad Recio (CCOO), Doña Miriam Hernando (CCOO), Don Antonio Herrera (CCOO)y a todos aquellos que participaron en el proyecto ( especialmente ): Doña Ana Cortés, Doña Fátima el Hachimi, Doña Yuli Fajardo y a todas las personas de la Asociación de Integración de la Comunidad Gitana de La Palma Palmilla.

\section{Biografia}

Gloria Santiago Méndez. Licenciada en Administración y Dirección de Empresas y Dra por la Universidad de Sevilla. Profesora asociada del departamento de Economía y Administración de empresas de la Universidad de Málaga. Principales áreas de trabajo, ventas/ marketing y voluntaria en temas sociales gloria.santiago@uma.es

Antonio Villanueva Martín. Licenciado en Derecho por la UNED, activista social, docente y filmmaker. Coordinador del Proyecto La Casa de Tod@s y responsable de la serie de cortometraje La trilogía de la Palmilla. antoniovillanuevamartin@gmail.com. 